\title{
溴化二甲基溴化锍催化合成二呋喃基芳基甲烷类化合物
}

\author{
刘巨艳* 黄海静焦德全 \\ (天津师范大学化学学院 天津市功能分子结构与性能重点实验室 无机-有机杂化功能材料化学 \\ 教育部重点实验室 天津 300387)
}

\begin{abstract}
摘要 室温无溶剂的条件下, 以澳化二甲基澳化锍(BDMS)为催化剂, 以 2-甲基呋喃和芳香醛为原料, 能高收率地合成 一系列二呋喃基芳基甲烷类化合物. 所有化合物结构通过 ${ }^{1} \mathrm{H} N M R 、{ }^{13} \mathrm{C} N M R$ 表征确定.

关键词＼cjkstart澳化二甲基溴化锍(BDMS); 二呋喃基芳基甲烷; 合成
\end{abstract}

\section{Synthesis of Difurylarylmethanes Catalyzed by Bromodimethylsulfonium Bromide}

\author{
Liu, Juyan* Huang, Haijing Jiao, Dequan \\ (Tianjin Key Laboratory of Structure and Performance for Functional Molecules, Key Laboratory of \\ Inorganic-Organic Hybrid Functional Materials Chemistry, Ministry of Education, \\ College of Chemistry, Tianjin Normal University, Tianjin 300387)
}

\begin{abstract}
A series of difurylarylmethanes were prepared in high yields by condensation of 2-methylfuran with aromatic aldehydes in the presence of bromodimethylsufonium bromide (BMDS) at room temperature under solvent-free conditions. All products were characterized by ${ }^{1} \mathrm{H}$ NMR and ${ }^{13} \mathrm{C}$ NMR spectra.

Keywords bromodimethylsufonium bromide (BMDS); difurylarylmethane; synthesis
\end{abstract}

呋喃代表了一类重要的分子结构, 这一结构广泛存 在于天然产物和药物分子之中(图 1) ${ }^{[1]}$, 多取代呋喃已经 被广泛用于医药化学 ${ }^{[2]} 、$ 材料化学 ${ }^{[3]} 、$ 农用化学品等 ${ }^{[4]}$ 方面. 带有不同取代基的呋喃化合物也是常用的有机合 成中间体 ${ }^{[5]}$, 因此，如何有效地合成多取代呋喃一直是 有机化学家关注的课题, 多年来也报道了一些有效的合 成方法 ${ }^{[6]}$.

近年来，一系列 2-甲基呋喃和芳香醛之间的反应揭 示了一种合成多取代呋喃的新策略 ${ }^{[7 \sim 13]}$. 到目前为止, 国内外文献报道的催化 2-甲基呋喃和芳香醛缩合的主 要催化剂有 $\mathrm{Yb}(\mathrm{OTf})_{3}{ }^{[7]} 、 \mathrm{AuCl}_{3}{ }^{[8]} 、 \mathrm{~B}\left(\mathrm{C}_{6} \mathrm{~F}_{5}\right)_{3}{ }^{[9]} 、 \mathrm{RuCl}_{3}$ • $3 \mathrm{H}_{2} \mathrm{O}^{[10]} 、 \mathrm{Hg}\left(\mathrm{ClO}_{4}\right)_{2} 、 \mathrm{Tl}\left(\mathrm{ClO}_{4}\right)_{3} 、 p-\mathrm{TSA}^{[11]} 、 \mathrm{I}_{2}{ }^{[12]}$ 、 $\mathrm{Bi}(\mathrm{OTf})_{3}{ }^{[13]}$. 尽管这些方法中有些具有很好的实用性, 但是其中有许多方法有严重的缺点, 例如, 使用昂贵的 和有毒的试剂, 较长的反应时间, 对湿度的敏感, 因此, 研究一种环保、高效的催化体系来实现 2-甲基呋喃和芳
香醛之间的缩合仍然具有很重要的意义.<smiles>COc1cc2c(c3oc(=O)c4c(c13)CCC4=O)[C@H]1C=CC[C@H]1O2</smiles>

Aflatoxin B1<smiles>Cc1ccc(CNc2ncnc3ccc(-c4ccc(C(=O)O)o4)cc23)o1</smiles>

NCGC00189258-01<smiles>O=C1c2cc(O)cc(O)c2C(=O)c2c1cc1c(c2O)[C@H]2C=CO[C@H]2O1</smiles>

Versicolorin A<smiles>O=Cc1cc(-c2ccc(O)c(O)c2)oc1-c1ccc(O)c(O)c1</smiles>

Dimethylfuroguaiacin
图 1 具有生物活性的呋喃衍生物

Figure 1 Furan derivatives with biological activities

\footnotetext{
* Corresponding author. E-mail: hxxyljy@mail.tjnu.edu.cn

Received December 2, 2016; revised February 20, 2017; published online March 3, 2017.

Project supported by the Natural Science Foundation of Tianjin City (No. 12JCZDJC34300).

天津市自然科学基金(No.12JCZDJC34300)资助项目.
} 
溴化二甲基溴化锍(BDMS) ${ }^{[14]}$ 是一种亮黄色粉末状固体, 制备简单、成本低. 它既可以作为催化剂催化多种化学 反应又可以作为溴化试剂参与反应, 且遇水容易分解, 因此后处理比较方便, 水洗就可以除去. 由于其高效的 催化活性和特有的化学性质 ${ }^{[15]}$, 已经引起大批科学家 的关注. 基于本课题组 ${ }^{[16]}$ 前期对溴化二甲基溴化锍催 化合成 $N$-取代吡咯类化合物、2-芳基苯并噻唑类化合物 及芳胺甲酰化反应方法的研究, 本工作以2-甲基呋喃和 芳香醛为底物, 通过溴化二甲基溴化锍催化高效、快速、 温和地实现了二呋喃基芳基甲烷类衍生物的合成(Eq. $1)$.

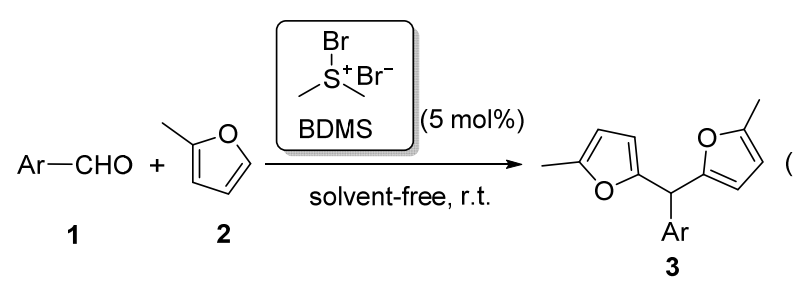

\section{1 结果与讨论}

\section{1 反应条件的优化}

为了确定最佳反应条件, 我们把 BDMS 催化苯甲 醛(1.0 mmol)和 2-甲基呋喃 $(2.4 \mathrm{mmol})$ 的反应作为模型 反应, 考察催化剂用量、反应介质对产品收率的影响, 实验结果见表 1. 我们通过观察模型反应在不同溶剂中 的产率情况, 发现用 $\mathrm{C}_{2} \mathrm{H}_{5} \mathrm{OH} 、$ EtOAc 、 $\mathrm{CH}_{3} \mathrm{CN}$ 、 $\mathrm{CH}_{3} \mathrm{COCH}_{3}$ 、四氢呋喃(THF)作溶剂进行该反应时, 都比 无溶剂反应时间长、反应产率低. 所以说明无溶剂条件 是最好的选择. 同时, 我们还发现催化剂的用量也影响 反应速率和收率. 在无溶剂条件下, 增加催化剂用量, 可以加快反应速度, 当催化剂的用量加至 $2 \%$ 时, 反应 $20 \mathrm{~min}$ 后, 产率达到 $80 \%$; 继续增加催化剂用量到 5 $\mathrm{mol} \%$, 反应 $2 \mathrm{~min}$ 后, 产率高达 $93 \%$; 但是, 继续增加 催化剂的用量, 产率不再升高. 因此, 无溶剂、催化剂用 量为 $5 \mathrm{~mol} \%$ 、反应温度为室温是最佳反应条件.

\section{2 反应底物的普适性研究}

在最优化的反应条件下, 为了探究该方法的普遍性 和适用范围, 使用各种芳香醛作为反应物, 在无溶剂条 件下, 与 2-甲基呋喃进行反应, 反应结果如表 2 所示. 从实验结果可以看出, 各种取代的芳香醛都能顺利与 2甲基呋喃进行缩合, 并以较高的产率得到目标化合物 (表 2, Entries 1 7,11,12). 芳环上的取代基的电子效应 对反应的影响较小, 不管是吸电子还是供电子的取代基 都能较高产率的得到目标化合物. 间位存在取代基的芳 香醛, 例如, 3-硝基苯甲醛和 3-甲氧基-4-羟基苯甲醛也
表 1 BDMS 催化合成二[(5-甲基)-2-呋喃基]苯基甲烷反应条 件优化 ${ }^{a}$

Table 1 Screening on the reaction conditions for the synthesis of bis(5-methyl-2-furyl)phenyl methane

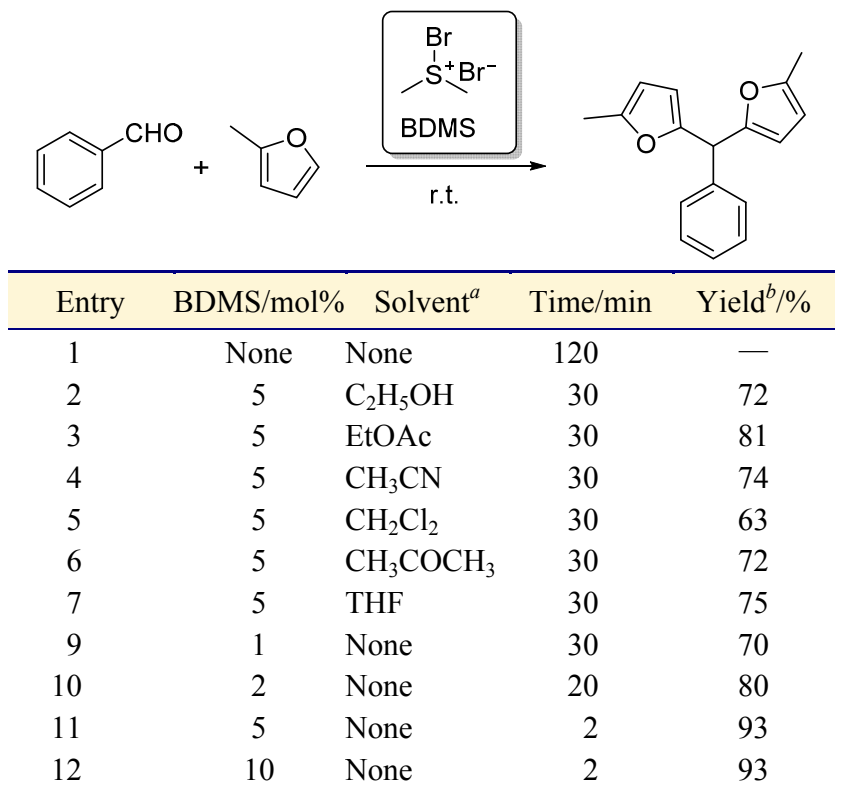

${ }^{a}$ All reactions were run with benzaldehyde $(1 \mathrm{mmol})$ and 2-methylfuran $(2.4$ $\mathrm{mmol})$ in the presence of BDMS in $4 \mathrm{~mL}$ of solvents at room temperature. ${ }^{b}$ Isolated yields.

能得到较高的收率(表 2, Entries 7,12). 此外, 杂环类化 合物 5-甲基呋喃-2-甲醛、噻吩-2-甲醛、吲哚- 3-甲醛也 是很好的底物, 它们与 2-甲基呋喃反应也能获得很好的 产率(表 2, Entries 8 10). 在研究中, 我们还发现在溴 化二甲基溴化锍存在下，3-甲硫基丙醛和柠檬醛不能与 2-甲基呋喃发生此类反应(表 2, Entries 13，14), 但造成 这一结果的具体原因尚不清楚.

与其他文献报道的催化剂在合成二呋喃基芳基甲 烷类化合物过程中的催化效果进行对比, 我们在表 3 中 列出了各种催化剂催化该反应所需要的催化剂当量、反 应时间、产物的产率. 与其他催化合成二呋喃基芳基甲 烷类反应的催化剂相比, 如 $\mathrm{Bi}(\mathrm{OTf})_{3}{ }^{[13]} 、 \mathrm{AuCl}_{3}{ }^{[8]}$ 、

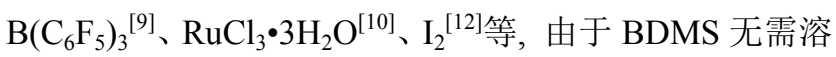
剂、室温条件下能迅速催化反应的进行, 因此 BDMS 的 催化效果更好. 且从表 3 不难看出, 用 BDMS 作催化剂, 比用已报道过的这些催化剂的反应产率高，反应时间 短，易于处理.

\section{3 反应机理的设想与讨论}

在前人研究 2-甲基呋喃与芳香醛类的反应的基础 上 $^{[7 \sim 13]}$, 我们提出了 BDMS 催化此类反应可能的反应机 理(Scheme 1). 首先, 芳香醛中的羰基与 BDMS 中带正 电的硫原子结合, 形成带有电正性的羰基碳正离子, 同 时, 2-甲基呋喃环的 $\alpha-\mathrm{C}$ 上的 $\mathrm{H}^{+}$与 BDMS 中的 $\mathrm{Br}^{-}$结合, 消除一分子 $\mathrm{HBr}$. 然后, 脱去 $\mathrm{H}^{+}$的 2-甲基呋喃进攻羰基 
表 2 无溶剂条件下 BDMS 催化 2-甲基呋喃与芳香醛类的反应 ${ }^{a}$

Table 2 BDMS-catalyzed reaction of 2-methylfuran with aryl aldehydes under solvent-free conditions

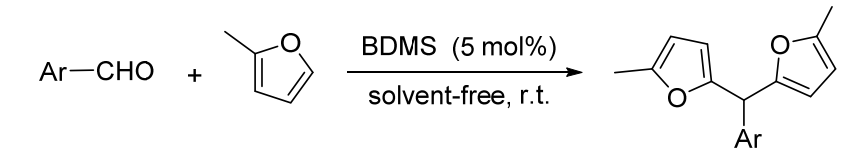

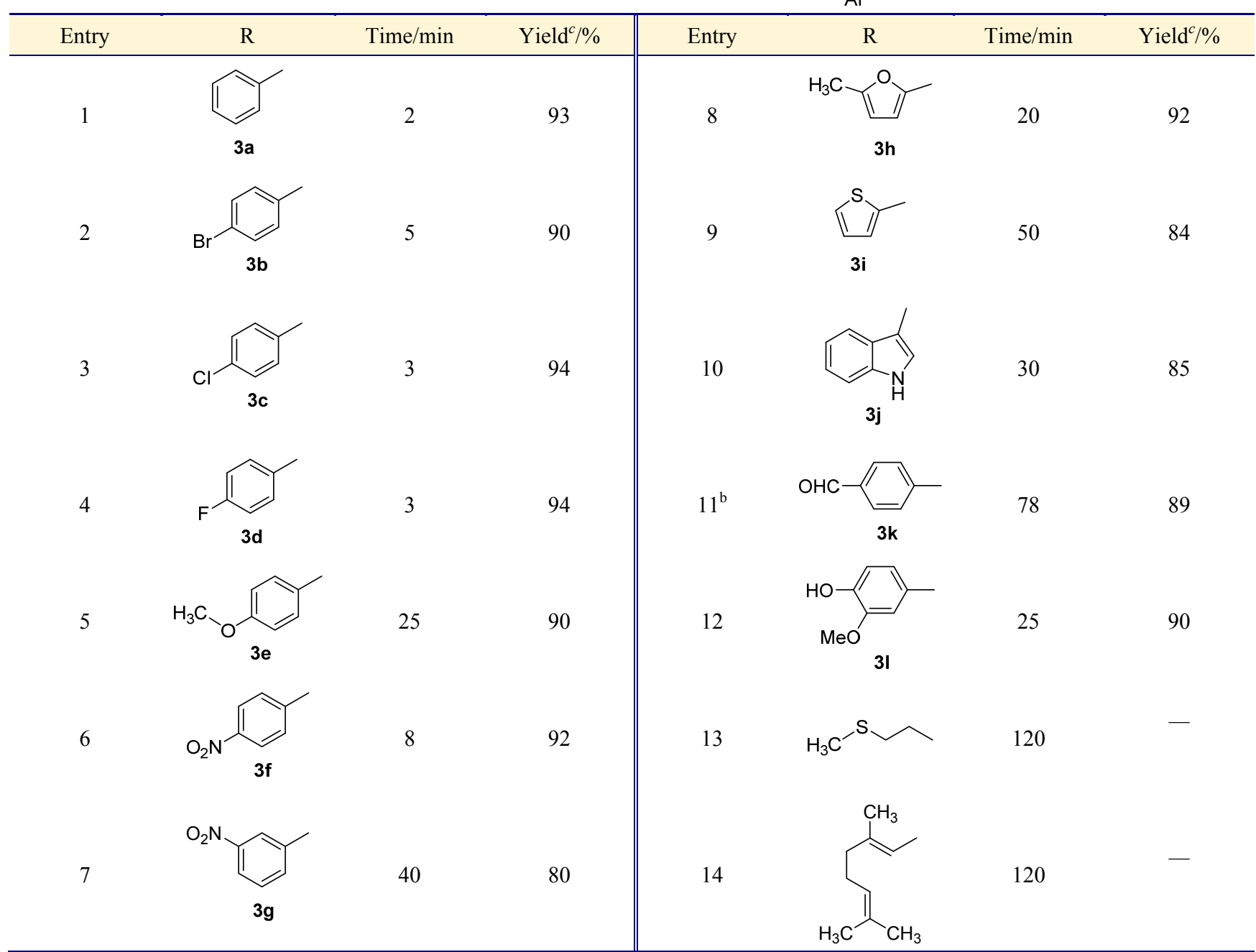

${ }^{a}$ General reaction conditions: aldehyde $(1.0 \mathrm{mmol}), 2$-methylfuran $(2.4 \mathrm{mmol})$, catalyst $(5 \mathrm{~mol} \%)$, room temperature. ${ }^{b}$ Reaction conditions: 1,4 -phthalaldehyde $(1.0$ mmol), 2-methylfuran ( $4.8 \mathrm{mmol})$, catalyst $(10 \mathrm{~mol} \%)$, room temperature. ${ }^{c}$ Isolated yields.

表 3 不同催化剂催化合成化合物 3a 效果对比

Table 3 Comparison of the effect of catalysts for the synthesis of $\mathbf{3 a}$

\begin{tabular}{|c|c|c|c|c|c|}
\hline Entry & Catalyst (mol/\%) & Conditions & Time/h & Yield $^{a} / \%$ & Ref. \\
\hline 1 & $\mathrm{Bi}(\mathrm{OTf})_{3}(5)$ & Toluene, r.t & 2 & 92 & [13] \\
\hline 4 & $\mathrm{AuCl}_{3}(1)$ & $\mathrm{CH}_{3} \mathrm{CN}$, r.t & 12 & 88 & [8b] \\
\hline 5 & $\mathrm{~B}\left(\mathrm{C}_{6} \mathrm{~F}_{5}\right)_{3}(5)$ & $\mathrm{CH}_{2} \mathrm{Cl}_{2}$, r.t & 0.25 & 92 & {$[9]$} \\
\hline 6 & $\mathrm{RuCl}_{3} \cdot 3 \mathrm{H}_{2} \mathrm{O}(5)$ & GDE, $5{ }^{\circ} \mathrm{C}$ & 144 & 79 & [10] \\
\hline 7 & $\mathrm{I}_{2}(10)$ & $\mathrm{H}_{2} \mathrm{O}$, r.t. & 72 & 79 & [12] \\
\hline
\end{tabular}

${ }^{a}$ Isolated yield.

碳正离子, 得到中间体 $\mathbf{a}$. 中间体 $\mathbf{a}$ 脱去一分子的 DMSO 得到中间体 $\mathbf{b}$. 反应过程中产生的 $\mathrm{Br}^{-}$继续与第 二分子的 2-甲基呋喃的 $\alpha-\mathrm{C}$ 上的 $\mathrm{H}^{+}$结合, 再消除一分 子 $\mathrm{HBr}$, 得到最终产物 3. 在这个反应过程中, BDMS 所 产生的 $\mathrm{HBr}$ 能够循环使用, 加速了反应进程.
催化量的 BDMS 在反应初期起到了活化羰基的作 用, 伴随着反应的进行, BDMS 逐渐转化成 $\mathrm{Me}_{2} \mathrm{SO}$ 和 $\mathrm{HBr}$ ，在随后的反应中，起催化作用的是 $\mathrm{HBr}$ 分子，为 了验证这个设想，我们以苯甲醛(1 mmol)和 2-甲基呋喃 $(2.4 \mathrm{mmol})$ 为底物, 在 $\mathrm{HBr}(\mathrm{g})(10 \mathrm{~mol} \%)$ 的作用下发生 


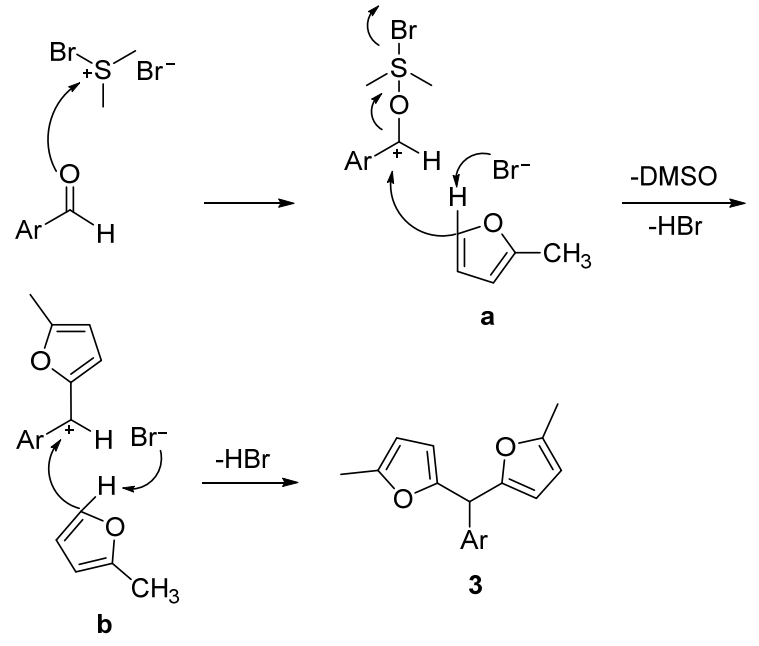

图式 1 BDMS 催化合成二呋喃基芳基甲烷类化合物可能的 反应机理

Scheme 1 Plausible mechanistic illustration of BDMS-catalyzed synthesis of difurylarylmethanes

反应，得到了预期的结果(Eq. 2).

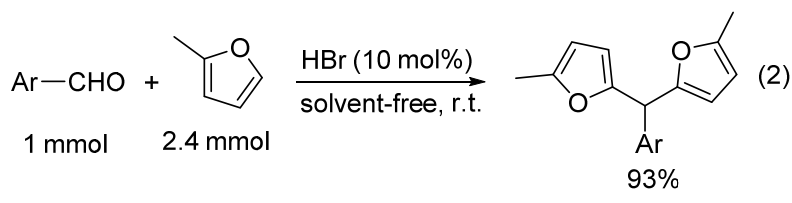

我们设想了 $\mathrm{HBr}$ 催化该反应的机理(Scheme 2), 在 反应过程中, $\mathrm{H}^{+}$起到了活化羰基的作用, 有利于 2-甲基 呋喃环的 $\alpha-\mathrm{C}$ 的亲核进攻, 通过 2-甲基呋喃环的 $\alpha-\mathrm{C}$ 的 两次亲核进攻, 最终脱去水分子和 $\mathrm{HBr}$ 分子, 使 $\mathrm{HBr}$ 分子能够被循环使用，最终在短时间内完成了反应.
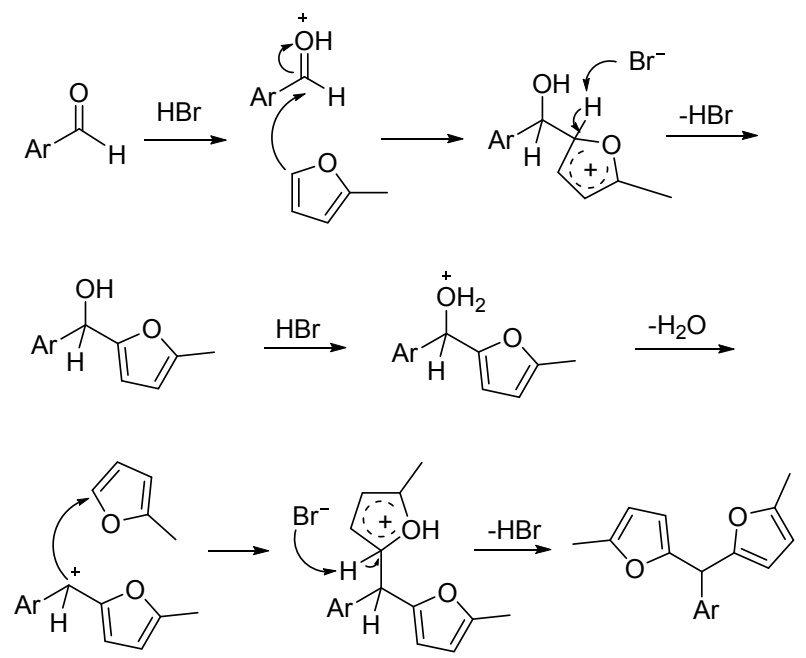

图式 $2 \mathrm{HBr}$ 催化合成二呋喃基芳基甲烷类化合物可能的反 应机理

Scheme 2 Plausible mechanistic illustration of HBr-catalyzed synthesis of difurylarylmethanes

\section{2 结论}

总之, 我们发现了一种在无机溶剂条件下使用 BDMS 作催化剂, 催化芳香醛与 2-甲基呋喃缩合合成二 呋喃基芳基甲烷类化合物的方法. 该方法具有操作简 便、产率高、反应时间短、过程环保等优点.

\section{3 实验部分}

\section{1 仪器与试剂}

核磁共振氢谱采用 BRUKER AC-P 300 型核磁共振 仪测定, 用 $\mathrm{CDCl}_{3}$ 为溶剂, TMS 为内标. 除特别说明外, 本文所用化学试剂均为市售化学纯或分析纯. 柱层析硅 胶(试剂级，200 300 目)购自青岛海洋化工有限公司， 直接使用. 所用的乙腈、二氯甲烷, 是加入适量的五氧 化二磷后回流，重蒸后使用. 所用乙醇、四氢呋喃用金 属钠回流重蒸后使用. 所用的乙酸乙酯、丙酮用分子篮 干燥，重蒸后使用. 所使用的 2-甲基呋喃和芳香醛均为 市售分析纯, 未加处理直接使用.

\section{2 二[(5-甲基)-2-呋喃基]苯基甲烷的制备}

在室温下，于 $25 \mathrm{~mL}$ 的圆底烧瓶中，加入 2-甲基呋 喃 $(2.4 \mathrm{mmol})$ 和芳香醛 $(1.0 \mathrm{mmol})$, 搅拌以使混合均匀, 加入溴化二甲基溴化锍(BDMS) $(0.05 \mathrm{mmol})$, 继续在室 温下搅拌适当时间, 用薄层层析色谱法(TLC)监测. 反 应完全后, 加入适量水, 搅拌, 加入适量乙酸乙酯萃取, 水洗 3 次, 分液, 收集有机相, 旋蒸, 除去溶剂的粗产品 运用柱层层析色谱法进行分离提纯, 得到纯净的二[(5甲基)-2-呋喃基]苯基甲烷(3a) ${ }^{[13]}$ : ${ }^{1} \mathrm{H}$ NMR $(400 \mathrm{MHz}$, $\mathrm{CDCl}_{3}$ ) $\delta: 7.35 \sim 7.28$ (br m, $5 \mathrm{H}$ ), 5.91 (br d, $J=4.3 \mathrm{~Hz}$, 4H), $5.37(\mathrm{~s}, 1 \mathrm{H}), 2.28(\mathrm{~s}, 6 \mathrm{H}) ;{ }^{13} \mathrm{C}$ NMR $(100 \mathrm{MHz}$, $\left.\mathrm{CDCl}_{3}\right) \delta: 152.8,151.4,140.0,128.39,128.36,126.9$, 108.1, 106.0, 45.1, 13.6.

二[(5-甲基)-2-呋喃基](4-溴苯基)甲烷 $(3 \mathbf{b}){ }^{[12]}:{ }^{1} \mathrm{H}$ NMR $\left(400 \mathrm{MHz}, \mathrm{CDCl}_{3}\right) \delta: 7.46(\mathrm{~d}, J=8.3 \mathrm{~Hz}, 2 \mathrm{H}), 7.15$ (d, $J=8.3 \mathrm{~Hz}, 2 \mathrm{H}$ ), 5.91 (br d, $J=3.0 \mathrm{~Hz}, 2 \mathrm{H}$ ), 5.90 (br d, $J=3.0 \mathrm{~Hz}, 2 \mathrm{H}), 5.31$ (s, 1H), 2.27 (s, 6H); ${ }^{13} \mathrm{C}$ NMR $(100$ $\left.\mathrm{MHz}, \mathrm{CDCl}_{3}\right) \delta: 152.1,151.6,139.1,131.5,130.1,120.9$, 108.3, 106.1, 44.5, 13.6 .

二[(5-甲基)-2-呋喃基] (4-氯苯基)甲烷 $(3 \mathbf{c c})^{[12]:}{ }^{1} \mathrm{H}$ NMR (400 MHz, $\left.\mathrm{CDCl}_{3}\right) \delta: 7.31(\mathrm{~d}, J=8.5 \mathrm{~Hz}, 2 \mathrm{H}), 7.21$ (d, $J=8.5 \mathrm{~Hz}, 2 \mathrm{H}$ ), 5.92 (br d, $J=3.1 \mathrm{~Hz}, 2 \mathrm{H}$ ), 5.90 (br d, $J=3.1 \mathrm{~Hz}, 2 \mathrm{H}), 5.33$ (s, 1H), 2.27 (s, 6H); ${ }^{13} \mathrm{C}$ NMR $(100$ $\left.\mathrm{MHz}, \mathrm{CDCl}_{3}\right) \delta: 152.5,151.9,138.9,133.1,130.1,128.9$, 108.6, 106.4, 44.8, 13.9.

二[(5-甲基)-2-呋喃基] (4-氟苯基)甲烷 $(3 \mathbf{d}){ }^{[12]:}{ }^{1} \mathrm{H}$ NMR (400 MHz, $\left.\mathrm{CDCl}_{3}\right) \delta: 7.24$ (dd, $\left.J=8.3,5.5 \mathrm{~Hz}, 2 \mathrm{H}\right)$, 
7.02 (t, $J=8.6 \mathrm{~Hz}, 2 \mathrm{H}), 5.91$ (br d, $J=2.7 \mathrm{~Hz}, 2 \mathrm{H}$ ), 5.89 (br d, $J=2.7 \mathrm{~Hz}, 2 \mathrm{H}), 5.34(\mathrm{~s}, 1 \mathrm{H}), 2.28(\mathrm{~s}, 6 \mathrm{H}) ;{ }^{13} \mathrm{C} \mathrm{NMR}$ $\left(100 \mathrm{MHz}, \mathrm{CDCl}_{3}\right) \delta: 161.9,152.6,151.6,135.7,129.9$, 115.2, 108.2, 106.1, 44.3, 13.6.

二[(5-甲基)-2-呋喃基](4-甲氧苯基)甲烷(3e $)^{[12]: ~}{ }^{1} \mathrm{H}$ NMR (400 MHz, $\mathrm{CDCl}_{3}$ ) $\delta: 7.21(\mathrm{~d}, J=8.7 \mathrm{~Hz}, 2 \mathrm{H}), 6.89$ (d, $J=8.7 \mathrm{~Hz}, 2 \mathrm{H}$ ), 5.91 (br d, $J=2.3 \mathrm{~Hz}, 2 \mathrm{H}$ ), 5.89 (br d, $J=2.3 \mathrm{~Hz}, 2 \mathrm{H}), 5.33$ (s, 1H), 3.82 (s, 3H), 2.28 (s, 6H); ${ }^{13} \mathrm{C}$ NMR (100 MHz, $\left.\mathrm{CDCl}_{3}\right) \delta: 158.5,153.1,151.3,132.1$, 129.3, 113.8, 107.9, 106.0, 55.2, 44.3, 13.6.

二[(5-甲基)-2-呋喃基](4-硝基苯基)甲烷(3f ${ }^{[12]}$ : ${ }^{1} \mathrm{H}$ NMR (400 MHz, $\left.\mathrm{CDCl}_{3}\right) \delta: 8.19(\mathrm{~d}, J=8.7 \mathrm{~Hz}, 2 \mathrm{H}), 7.43$ (d, $J=8.7 \mathrm{~Hz}, 2 \mathrm{H}$ ), 5.96 (br d, $J=3.0 \mathrm{~Hz}, 2 \mathrm{H}$ ), 5.94 (br d, $J=3.0 \mathrm{~Hz}, 2 \mathrm{H}), 5.45$ (s, 1H), 2.27 (s, 6H); ${ }^{13} \mathrm{C}$ NMR $(100$ $\left.\mathrm{MHz}, \mathrm{CDCl}_{3}\right) \delta: 152.5,151.4,147.9,147.5,129.7,124.2$, 109.3, 106.7, 45.3, 14.0.

二 [(5- 甲基)-2-呋喃基](3-硝基苯基)甲烷 $(\mathbf{3 g})^{[7]}$ : ${ }^{1} \mathrm{HNMR}\left(400 \mathrm{MHz}, \mathrm{CDCl}_{3}\right.$ ) $\delta: 8.13 \sim 8.11(\mathrm{~m}, 2 \mathrm{H}), 7.59$ $(\mathrm{d}, J=8 \mathrm{~Hz}, 2 \mathrm{H}), 7.50 \sim 7.46(\mathrm{~m}, 1 \mathrm{H}), 5.94 \sim 5.92(\mathrm{~m}, 4 \mathrm{H})$, $5.44(\mathrm{~s}, 1 \mathrm{H}), 2.25(\mathrm{~s}, 6 \mathrm{H}) ;{ }^{13} \mathrm{C}$ NMR $\left(100 \mathrm{MHz}, \mathrm{CDCl}_{3}\right) \delta$ : $151.9,151.0,148.3,142.1,134.4,129.1,123.2,121.9$, 108.6, 106.1, 44.5, 13.4.

2-[二(5-甲基呋喃-2-基)甲基]-5-甲基呋喃(3h) ${ }^{[11]}:{ }^{1} \mathrm{H}$ NMR (300 MHz, $\left.\mathrm{CDCl}_{3}\right) \delta: 2.26(\mathrm{~s}, 9 \mathrm{H}), 5.33(\mathrm{~s}, 1 \mathrm{H}), 5.87$ $(\mathrm{s}, 3 \mathrm{H}), 5.93(\mathrm{~s}, 3 \mathrm{H}) ;{ }^{13} \mathrm{CNMR}\left(75 \mathrm{MHz}, \mathrm{CDCl}_{3}\right) \delta: 13.6$, 39.3, 106.4, 107.9, 150.8, 151.3.

二[(5-甲基)-2-呋喃基](2-噻吩基)甲烷 $(3 \mathbf{i})^{[8 \mathrm{a}]}$ : ${ }^{1} \mathrm{H}$ NMR $\left(300 \mathrm{MHz}, \mathrm{CDCl}_{3}\right) \delta: 2.26(\mathrm{~s}, 6 \mathrm{H}), 5.56(\mathrm{~s}, 1 \mathrm{H}), 5.87$ $(\mathrm{s}, 2 \mathrm{H}), 5.95(\mathrm{~s}, 2 \mathrm{H}), 6.87 \sim 6.93(\mathrm{~m}, 2 \mathrm{H}), 7.17(\mathrm{~d}, 1 \mathrm{H}, J=$ $4.9 \mathrm{~Hz}) ;{ }^{13} \mathrm{C} \mathrm{NMR}\left(75 \mathrm{MHz}, \mathrm{CDCl}_{3}\right) \delta: 40.4,106.4,108.1$, 124.7, 125.8, 126.7, 143.4, 151.6, 152.4.

3-[二(5-甲基-2-呋喃基)甲基]-1H-吲哚(3j) ${ }^{[8 \mathrm{a}]}$ : ${ }^{1} \mathrm{H}$ NMR (300 MHz, $\left.\mathrm{CDCl}_{3}\right) \delta: 2.25(\mathrm{~s}, 6 \mathrm{H}), 5.58(\mathrm{~s}, 1 \mathrm{H}), 5.85$ $(\mathrm{s}, 2 \mathrm{H}), 5.89(\mathrm{~s}, 2 \mathrm{H}), 6.93(\mathrm{~s}, 1 \mathrm{H}), 7.03$ (uneven triplet, $1 \mathrm{H}$, $J=7.6,7.3 \mathrm{~Hz}$ ), 7.14 (uneven triplet, $1 \mathrm{H}, J=7.9,7.1 \mathrm{~Hz}$ ), 7.28 (d, $J=8.1 \mathrm{~Hz}, 1 \mathrm{H}), 7.47$ (d, $J=7.9 \mathrm{~Hz}, 1 \mathrm{H}), 7.91$ (s, $1 \mathrm{H}) ;{ }^{13} \mathrm{C} \mathrm{NMR}\left(75 \mathrm{MHz}, \mathrm{CDCl}_{3}\right) \delta: 13.7,36.8,106.1$, $107.5,111.1,115.1,119.4,119.6,122.0,122.9,126.5$, $136.4,150.9,153.0$.

四[(5-甲基)-2-呋喃基]-1，4-二次甲基苯(3k $)^{[8 \mathrm{a}]}$ ： ${ }^{1} \mathrm{H}$ NMR (300 MHz, $\left.\mathrm{CDCl}_{3}\right) \delta: 2.24(\mathrm{~s}, 12 \mathrm{H}), 5.30(\mathrm{~s}, 2 \mathrm{H})$, $5.86(\mathrm{~s}, 8 \mathrm{H}), 7.17(\mathrm{~s}, 4 \mathrm{H}) ;{ }^{13} \mathrm{C}$ NMR $\left(75 \mathrm{MHz}, \mathrm{CDCl}_{3}\right) \delta$ : 13.6, 44.7, 106.0, 108.1, 128.4, 138.6, 151.3, 152.8.

二[(5-甲基)-2-呋喃基](4-羟基-3-甲氧苯基)甲烷 $(3 \mathrm{I}){ }^{[12]}:{ }^{1} \mathrm{H}$ NMR $\left(400 \mathrm{MHz}, \mathrm{CDCl}_{3}\right) \delta: 6.88(\mathrm{~d}, J=8.1 \mathrm{~Hz}$,
$1 \mathrm{H}), 6.80 \sim 6.75(\mathrm{~m}, 2 \mathrm{H}), 5.90(\mathrm{br} \mathrm{d}, J=2.8 \mathrm{~Hz}, 2 \mathrm{H}), 5.88$ (br d, $J=2.8 \mathrm{~Hz}), 5.56(\mathrm{~s}, 1 \mathrm{H}), 5.28(\mathrm{~s}, 1 \mathrm{H}), 3.86(\mathrm{~s}, 3 \mathrm{H})$, $2.27(\mathrm{~s}, 6 \mathrm{H}) ;{ }^{13} \mathrm{C}$ NMR $\left(100 \mathrm{MHz}, \mathrm{CDCl}_{3}\right) \delta: 153.2,151.4$, $146.6,144.7,132.0,121.3,114.4,111.2,108.1,106.2$, $55.9,44.9,13.6$.

辅助材料(Supporting Information) 化合物的 ${ }^{1} \mathrm{H}$ NMR 和 ${ }^{13} \mathrm{C} \mathrm{NMR}$ 谱图. 这些材料可以免费从本刊网站(http:// sioc-journal.cn/)上下载.

\section{Referenes}

[1] (a) Sperry, J. B.; Wright, D. L. Curr. Opin. Drug Discovery Dev. 2005, 8,723

(b) Boto, A.; Alvarez, L. In Heterocycles in Natural Product Synthesis, Wiley-VCH Verlag GmbH \& Co. KGaA, 2011, p. 97.

(c) Ye, O. Z.; Xie, S. X.; Huang, M.; Huang, W. J.; Lu, J. P.; Ma, Z. Q. J. Am. Chem.Soc. 2004, 126, 13940.

(d)Tan, H. B.; Chen, X. Z.; Liu, Z.; Wang, D. Z. Tetrahedron 2012 , $68,3952$.

[2] (a) Cui, Z. N.; Shi, Y. X.; Cui, J. R.; Ling, Y.; Li, B. J.; Yang, X. L. Chem. Biol. Drug Des. 2012, 79, 121.

(b) Iyer, R. S.; Coles, B. F.; Raney, K. D.; Thier, R.; Guengerich, F. P.; Harris, T. M. J. Am. Chem. Soc. 1994, 116, 160.

(c) Bren, U.; Guengerich, F. P.; Mavri, J. Chem. Res. Toxicol. 2007, 20, 1134.

(d) Jubert, C.; Mata, J.; Bench, G.; Dashwood, R.; Pereira, C.; Tracewell, W.; Turteltaub, K.; Williams, D.; Bailey, G. Cancer Prev. Res. 2009, 2, 1015 .

[3] (a) Li, B. L. Chin. J. Org. Chem. 2015, 35, 2487. (b) Pan, X. X.; Huo, L. J. Chin. J. Org. Chem. 2016, 36, 687

[4] (a) Rodricks, J. V. J. Agric. Food Chem. 1969, 17, 457.

(b) Lee, N. A.; Wang, S.; Allan, R. D.; Kennedy, I. R. J. Agric. Food Chem. 2004, 52, 2746

[5] (a) Lipshutz, B. H. Chem. Rev. 1986, 86, 795.

(b) Wong, H. N. C.; Yang, Y. Tetrahedron 1994, 50, 9583.

(c) Lee, H.-K.; Chan, K.-F.; Hui, C.-W.; Yim, H.-K.; Wu, X.-W.; Wong, H. N. C. Pure Appl. Chem. 2005, 77, 139.

[6] (a) Gulevich, A. V.; Dudnik, A. S.; Chernyak, N.; Gevorgyan, V. Chem. Rev. 2013, 113, 3084.

(b) Keay, B. A. Chem. Soc. Rev. 1999, 28, 209

(c) Ma, S. Acc. Chem. Res. 2003, 36, 701.

(d) Zou, W.; He, Z. R.; He, Z. J. Chin. J. Org. Chem. 2015, 35, 1739.

[7] Genovese, S.; Epifano, F.; Pelucchini, C.; Curini, M. Eur. J. Org Chem. 2009, 2009, 1132 .

[8] (a) Nair, V.; Abhilash, K. G.; Vidya, N. Org. Lett. 2005, 7, 5857. (b) Nair, V.; Vidya, N.; Abhilash, K. G. Synthesis 2006, 21, 3647.

[9] Chandrasekhar, S.; Khatun, S.; Rajesh, G.; Reddy, Ch. J. Tetrahedron Lett 2009, 50, 669.

[10] Qu, H. E.; Xiao, C.; Wang, N.; Yu, K. H.; Hu, Q. S.; Liu, L. X. Molecules 2011, 16, 3855.

[11] Hashmi, A. S. K.; Schwarz, L.; Rubenbauer, P.; Blanco, M. C. $A d v$. Synth. Catal. 2006, 348, 705.

[12] Jaratjaroonphong, J.; Tuengpanya, S.; Saeeng, R.; Udompong, S.; Srisook, K. Eur. J. Med. Chem.. 2014, 83, 561.

[13] Jaratjaroonphong, J.; Tuengpanya, S.; Ruengsangtongkul, S. J. Org. Chem. 2015, 80, 559 .

[14] (a) Choudhury, L. H.; Pravin, T.; Khan, A. T. Tetrahedron 2009, 65, 9513.

(b) Olah, G. A.; Vankar, Y. D.; Arvanaghi, M.; Surya Prakash, G. K. Synthesis 1979, 720.

(c) Furukawa, N.; Inoue, T.; Aida, T.; Oae, S. J. Chem. Soc., Chem. Commun. 1973, 212a. 
[15] (a) Choudhury, L. H. Synlett 2006, 1619.

(b) Khan, A. T.; Ali, A. M.; Goswami, P.; Choudhury, L. H. J. Org. Chem. 2006, 71, 8961.

(c) Das, B.; Krishnaiah, M.; Katta, V. Tetrahedron Lett. 2006, 47, 4457.

(d) Khan, A. T.; Mondal, E.; Borah, B. M.; Ghosh, S. Eur. J. Org. Chem. 2003, 4113.

(e) Khan, A. T.; Islam, S.; Majee, A.; Chattopadhyay, T.; Ghosh, S. J. Mol. Catal., A: Chem. 2005, 239, 158.

(f) Khan, A. T.; Sahu, P. R.; Majee, A. J. Mol. Catal., A: Chem. 2005, 226, 207.

(g) Khan, A. T.; Mondal, E.; Ghosh, S.; Islam, S. Eur. J. Org.
Chem. 2004, 2002.

(h) Das, B.; Holla, H.; Srinivas, Y. Tetrahedron Lett. 2007, 48, 61.

[16] (a) Zhao, C. Y.; Liu, J. Y.; Wang, Y.; Zhao, X. J.; Yuan, B.; Yue, M. M. Synth. Commun. 2014, 44, 827.

(b) Yue, M. M.; Liu, J. Y.; Wang, Y.; Yuan, B. Chin. J. Org. Chem. 2014, 34, 190 .

(c) Zhang, L. J.; Liu, J. Y.; Wang, Y.; Chin. J. Org. Chem. 2013, 33, 339.

(d) Huang, H. J.; Liu, J. Y.; Ma, E. Z.; Cao, Y. Y. Chin. J. Org. Chem. 2015, 35, 2372 .

(Li, L.; Fa, Y.) 